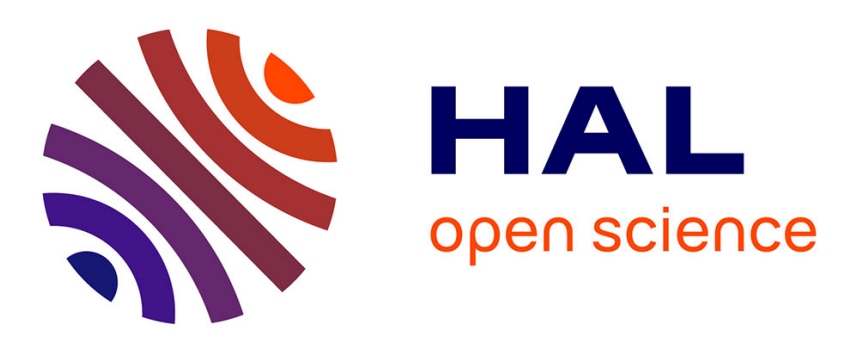

\title{
Conservation laws and prediction methods for stress concentration fields
}

\author{
Holm Altenbach, Victor A. Eremeyev, Andreas Kutschke, Konstantin \\ Naumenko
}

\section{- To cite this version:}

Holm Altenbach, Victor A. Eremeyev, Andreas Kutschke, Konstantin Naumenko. Conservation laws and prediction methods for stress concentration fields. Acta Mechanica, 2011, 218 (3-4), pp.349-355. hal-00823987

\section{HAL Id: hal-00823987 \\ https://hal.science/hal-00823987}

Submitted on 20 May 2013

HAL is a multi-disciplinary open access archive for the deposit and dissemination of scientific research documents, whether they are published or not. The documents may come from teaching and research institutions in France or abroad, or from public or private research centers.
L'archive ouverte pluridisciplinaire HAL, est destinée au dépôt et à la diffusion de documents scientifiques de niveau recherche, publiés ou non, émanant des établissements d'enseignement et de recherche français ou étrangers, des laboratoires publics ou privés. 


\title{
H. Altenbach • V. A. Eremeyev • A. Kutschke • K. Naumenko \\ Conservation laws and prediction methods for stress concentration fields
}

\begin{abstract}
Starting with the Eshelby-type conservation law, path-independent line and surface integrals that allow the comparison of averaged strain-energy densities in the notch area for linear elastic and non-linear elastic material behaviors are derived. The analysis shows that a point (two-dimensional problems) and a curve (three-dimensional problems) exist on the notch boundary, where the values of the strain-energy densities are almost the same. The conditions are discussed, for which the equality of the strain energies is guaranteed. The theoretical results are illustrated by two finite-element examples.
\end{abstract}

\section{Introduction}

An essential step in the general structural analysis is the estimation of localized stress, strain, and damage fields in notches, holes, grooves, welds, and other stress concentration zones. To compute such fields, an inelastic non-linear finite-element analysis is required. Although comprehensive inelastic constitutive models that reflect hardening/recovery, softening, damage, and other processes exist, e.g., [1-3], their calibration with experimental data and the utilization within the finite-element code are usually expensive. Furthermore, the inelastic structural analysis over many cycles of non-stationary loading are time consuming, if at all possible. Therefore, several rules were developed that allow a fast estimation of localized inelastic fields based on the corresponding linear elastic computations and idealized inelastic material laws. Examples include the Neuber rule [4] and the Glinka rule [5] that are widely used in engineering practice, for example in design, see [6,7] among others.

In this paper, we discuss the theoretical background for the derivation of simplified methods to predict multiaxial fields in the neighborhood of stress concentration zones. Starting with Eshelby-type conservation laws, e.g., [8-10], we derive path-independent surface integrals that allow the comparison of averaged strainenergy densities in the notch area for linear elastic and non-linear elastic material behaviors. The aims of the paper can be summarized as follows:

- The standard techniques to verify both the Neuber and the Glinka methods are usually based on the J-integral, e.g., [7], and, therefore, limited to plane stress/strain situations. By the use of a general conservation law, an extension to three-dimensional stress concentration problems is given.

- It will be shown that a point (in the two-dimensional case) or a curve (in the three-dimensional case) on the notch surface exists, where the actual strain-energy density of non-linear elastic (elastic-plastic) material almost coincides with the strain-energy density of the linear elastic comparison solution.

Dedicated to our colleague and friend Prof. Dr.-Ing. Reinhold Kienzler in occasion of his sixtieth birthday.

H. Altenbach $(\bowtie) \cdot$ V. A. Eremeyev $\cdot$ A. Kutschke $\cdot$ K. Naumenko

Center of Engineering Sciences, Martin-Luther-Universität Halle-Wittenberg, 06099 Halle (Saale), Germany 
- We formulate conditions for which the equality of the strain-energy densities is guaranteed.

- Two numerical examples are presented to illustrate the theoretical analysis.

\section{Energy-density estimates}

Let us assume that a non-linear elastic body occupies the volume $V$ with the smooth surface $A$. Neglecting body forces, the Lagrangian equilibrium equations and the static boundary conditions have the form

$$
\nabla \cdot \sigma=0,\left.\quad n \cdot \sigma\right|_{A_{f}}=0,\left.\quad n \cdot \sigma\right|_{A_{t}}=\boldsymbol{t},\left.\quad u\right|_{A_{u}}=0
$$

where $\sigma$ is the stress tensor, $\boldsymbol{u}$ the displacement vector, $\nabla$ the nabla (Hamilton) operator, $\boldsymbol{n}$ the unit-normal vector to the body surface $A=A_{u} \cup A_{f} \cup A_{t}$. The part $A_{u}$ is clamped, $A_{f}$ is traction-free, while the surface loads $t$ act on the remaining part $A_{t}$, Fig. 1 .

For homogeneous materials, the following conservation law is established [8]:

$$
\nabla \cdot \boldsymbol{b}=0, \quad \boldsymbol{b}=W I-\sigma \cdot(\nabla u)^{T}
$$

where $\boldsymbol{b}$ is the Eshelby tensor, $W$ the strain-energy function, $I$ the unit tensor, and the superscript $T$ denotes the transpose of a second-order tensor.

Integrating Eq. (2.1) over any part $V_{*} \subset V$ with the boundary $A_{*}$ and applying the Gauss-Ostrogradsky theorem, we obtain the identity

$$
\iint_{A_{*}} \boldsymbol{n} \cdot \boldsymbol{b} \mathrm{d} A=0 .
$$

Equation (3) may be used for the construction of the path-independent surface and line integrals, see [8] for details.

Let us consider the second boundary-value problem for the body with the same shape and the same boundary conditions but made from a linear elastic material. We assume that the corresponding constitutive equation is the limit case of the non-linear constitutive equation for small strains, that is, for this linear elastic material, the following relations hold true:

$$
\sigma \approx \sigma_{\circ}, \quad W \approx W_{\circ}
$$

when $\|\nabla \boldsymbol{u}\| \ll 1$. In (4), $\sigma_{\circ}$ and $W_{\circ}$ denote the stress tensor and the strain-energy density for the linear elastic body, respectively. Let us designate this body as the comparison body. In other words, the comparison body occupies the same volume $V$ with the boundary $A$, while its properties are described by Hooke's law. The boundary-value problem for the comparison body has the form

$$
\nabla \cdot \sigma_{\circ}=\mathbf{0},\left.\quad n \cdot \boldsymbol{\sigma}_{\circ}\right|_{A_{f}}=\mathbf{0},\left.\quad \boldsymbol{n} \cdot \boldsymbol{\sigma}_{\circ}\right|_{A_{t}}=\boldsymbol{t},\left.\quad u\right|_{A_{u}}=\mathbf{0} .
$$

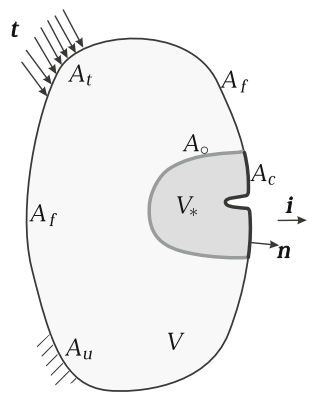

Fig. 1 A body with a notch. The gray region $V_{*}$ denotes the influence zone of the non-linear effect. The white region $V \backslash V_{*}$ corresponds to the zone where the difference between solutions of the non-linear and linear problems can be neglected 
Then, the conservation law and the integral identity are given by

$$
\begin{gathered}
\nabla \cdot \boldsymbol{b}_{\circ}=\mathbf{0}, \quad \boldsymbol{b}_{\circ}=W_{\circ} \boldsymbol{I}-\boldsymbol{\sigma}_{\circ} \cdot(\nabla \boldsymbol{u})^{T}, \\
\iint_{A_{*}} \boldsymbol{n} \cdot \boldsymbol{b}_{\circ} \mathrm{d} A=0 .
\end{gathered}
$$

As an example of a non-linear constitutive law, let us consider the Ramberg-Osgood model

$$
\begin{aligned}
E \varepsilon & =(1+v) \operatorname{dev} \sigma-(1-2 v) \sigma_{\mathrm{m}} \boldsymbol{I}+\frac{3}{2} \alpha\left(\frac{\sigma_{\mathrm{vM}}}{\sigma_{\mathrm{y}}}\right)^{n-1} \operatorname{dev} \boldsymbol{\sigma}, \\
\varepsilon & =\frac{1}{2}\left[\nabla \boldsymbol{u}+(\nabla u)^{T}\right], \\
\sigma_{\mathrm{m}} & =-\frac{1}{3} \operatorname{tr} \sigma, \quad \sigma_{\mathrm{vM}}^{2}=\frac{3}{2} \operatorname{tr}(\operatorname{dev} \sigma)^{2} .
\end{aligned}
$$

Here, $E$ and $v$ are Young's modulus and Poisson's ratio, respectively, $\varepsilon$ is the linear strain tensor, $\alpha$ the yield offset, $\sigma_{\mathrm{y}}$ the yield stress, $n$ the hardening exponent, $\operatorname{tr}$ and dev are the trace and deviator operators, and $\sigma_{\mathrm{vM}}$ and $\sigma_{\mathrm{m}}$ denote the von Mises stress and the hydrostatic stress, respectively. The Ramberg-Osgood model describes the non-linear relationship between stress and strain tensors for plastic materials during the loading.

The corresponding constitutive equation for the comparison body is given by Hooke's law

$$
E \varepsilon=(1+v) \operatorname{dev} \sigma_{\circ}+\frac{(1-2 v)}{3} I \operatorname{tr} \sigma_{\circ}
$$

In what follows, we use two assumptions:

1. Under the applied traction $t$, there are stress concentration zones such as notches, holes, etc. The surface of these zones is traction-free.

2. The non-linear material behavior is localized in the vicinity of the stress concentration zone (gray region in Fig. 1), i.e., far from this zone, the material behavior is linear.

More precisely, we assume the existence of the surface $A_{\circ} \subset V$ surrounding the concentration zone where the stress and the strain fields of the non-linear body which satisfy (1) almost coincide with the solution of the boundary-value problem (5) for the comparison body.

Consider the part $V_{*} \subset V$ containing the concentration zone with the boundary $A_{*}=\partial V_{*}$ that consists of the surface $A_{\circ}$ and the part of the free boundary $A_{c} \subset A_{f}, A_{*}=A_{\circ} \cup A_{c}$, Fig. 1. Applying Eqs. (3) and (7) to this surface, we obtain

$$
\begin{aligned}
& \iint_{A_{c}} n \cdot \boldsymbol{b} \mathrm{d} A+\iint_{A_{\circ}} \boldsymbol{n} \cdot \boldsymbol{b} \mathrm{d} A=\mathbf{0}, \\
& \iint_{A_{c}} n \cdot b_{\circ} \mathrm{d} A+\iint_{A_{\circ}} n \cdot b_{\circ} \mathrm{d} A=0 .
\end{aligned}
$$

By definition of $A_{\circ}$, it follows that $b \approx b_{\circ}$ on $A_{\circ}$. Hence, from (10), we obtain the relation

$$
\iint_{A_{c}} \boldsymbol{n} \cdot\left(\boldsymbol{b}-b_{\circ}\right) \mathrm{d} A \approx 0
$$

Using the boundary conditions

$$
\left.\boldsymbol{n} \cdot \boldsymbol{\sigma}\right|_{A_{c}}=\mathbf{0},\left.\quad n \cdot \boldsymbol{\sigma}_{\circ}\right|_{A_{c}}=0,
$$

and Eq. (11), the following integral relation for the strain-energy functions $W$ and $W_{\circ}$ can be derived:

$$
\iint_{A_{C}}\left(W-W_{\circ}\right) n \mathrm{~d} A \approx 0 .
$$

The scalar product of Eq. (12) with any constant vector $i$ results in the scalar equation

$$
\iint_{A_{c}}\left(W-W_{\circ}\right) n_{i} \mathrm{~d} A \approx 0 .
$$

where $n_{i}=n \cdot \boldsymbol{i}$. 
If $n_{i} \neq 0$, then from (12) or (13), it follows that at least one curve $L \subset A_{c}$ exists, where $W=W_{\circ}$. Indeed, Eq. (13) implies that the integrand should change its sign in some points of $A_{c}$. Therefore, $\left(W-W_{\circ}\right) n_{i}=0$ in that points. The continuity condition of the integrand requires that these points form at least one curve $L$.

For plane problems, the surface integrals reduce to line integrals, i.e., to J-integrals. Let us consider the plane stress (strain) problems for a U-notch. Then, Eq. (13) transforms to

$$
\int_{\Gamma_{c}}\left(W-W_{\circ}\right) n_{i} \mathrm{~d} s \approx 0
$$

where $\Gamma_{c}$ is the curve describing the notch. From Eq. (14), it follows that at least one point exists along $\Gamma_{c}$ such that $W=W_{\circ}$.

\section{Example for U-notched plate}

To illustrate our results, let us consider an example for a U-notched rectangular plate subjected to uniform traction $t$ under plane stress conditions, Fig. 2.

Let us select the contour $\Gamma=\mathrm{ABCDEF}$ for the quarter of the plate as the integration path. Then, Eq. (3) can be transformed to

$$
\begin{aligned}
\int_{\Gamma} \boldsymbol{n} \cdot \boldsymbol{b} \cdot \boldsymbol{i} \mathrm{d} s \equiv & \int_{\mathrm{AB}} \boldsymbol{n} \cdot \boldsymbol{b} \cdot \boldsymbol{i} \mathrm{d} s+\int_{\mathrm{BC}} \boldsymbol{n} \cdot \boldsymbol{b} \cdot \boldsymbol{i} \mathrm{d} s+\int_{\mathrm{CD}} \boldsymbol{n} \cdot \boldsymbol{b} \cdot \boldsymbol{i} \mathrm{d} s \\
& +\int_{\mathrm{DE}} \boldsymbol{n} \cdot \boldsymbol{b} \cdot \boldsymbol{i} \mathrm{d} s+\int_{\mathrm{EF}} \boldsymbol{n} \cdot \boldsymbol{b} \cdot \boldsymbol{i} \mathrm{d} s+\int_{\mathrm{FA}} \boldsymbol{n} \cdot \boldsymbol{b} \cdot \boldsymbol{i} d s=0 .
\end{aligned}
$$

From the symmetry of the boundary-value problem, it follows that $\boldsymbol{n} \cdot \boldsymbol{b} \cdot \boldsymbol{i}=0$ at $B C$. Furthermore, $n \cdot b \cdot i=-W$ for the path $A B, n \cdot b \cdot i=W n_{i}$ for the path $C D, n \cdot b \cdot i=0$ for the path $D E, n \cdot b \cdot i=W$ for the path $E F$, and $n \cdot b \cdot \boldsymbol{i}=t \cdot(\nabla u)^{T} \cdot \boldsymbol{i}$ along $E A$. As a result, we have

$$
-\int_{\mathrm{AB}} W \mathrm{~d} s+\int_{\mathrm{CD}} W n_{i} \mathrm{~d} s+\int_{\mathrm{EF}} W \mathrm{~d} s+\int_{\mathrm{FA}} t \cdot(\nabla u)^{T} \cdot \boldsymbol{i} \mathrm{d} s=0 .
$$

For the comparison body, we obtain the similar equation

$$
-\int_{\mathrm{AB}} W_{\circ} \mathrm{d} s+\int_{\mathrm{CD}} W_{\circ} n_{i} \mathrm{~d} s+\int_{\mathrm{EF}} W_{\circ} \mathrm{d} s+\int_{\mathrm{FA}} \boldsymbol{t} \cdot\left(\nabla \boldsymbol{u}_{\circ}\right)^{T} \cdot \boldsymbol{i} \mathrm{d} s=0 .
$$

If the non-linear effects are localized near $C D$, then $W \approx W_{\circ}$ along $A B$ and $E F$ and $\boldsymbol{u} \approx \boldsymbol{u}_{\circ}$ along $F A$. By subtracting Eq. (17) from Eq. (16), we obtain

$$
\int_{\mathrm{CD}} W n_{i} \mathrm{~d} s-\int_{\mathrm{CD}} W_{\circ} n_{i} \mathrm{~d} s \approx 0 .
$$

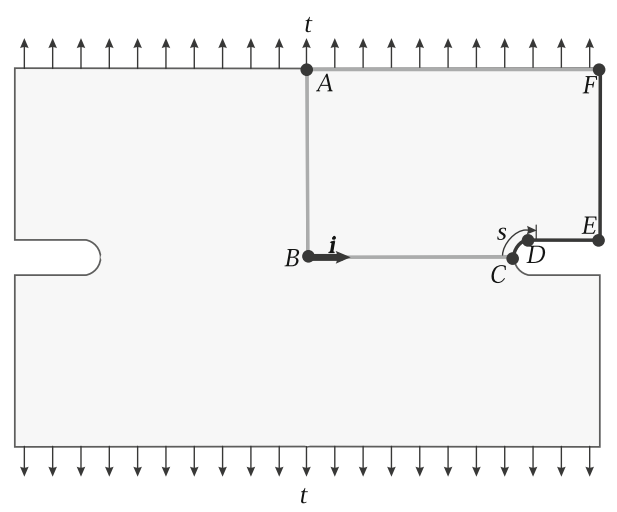

Fig. 2 Plate with two U-notches 
Equation (18) illustrates the general integral equation (14) for the U-notched plate with $\Gamma_{c}=C D$. Thus, at least one point on $C D$ exists where $W=W_{\circ}$.

To examine our conclusions, let us present two numerical examples for a U-notched rectangular plate with different geometries. The shape and size of the U-notch are assumed to be the same, while the length and width of the plates are different. The dimensions of the plates $(\mathrm{mm})$, the loading, and the boundary conditions are presented in Fig. 3. To estimate the energy densities, the finite-element analyses for both plate geometries are performed using the ABAQUS code with the following material parameters for the Ramberg-Osgood model:

$$
\begin{aligned}
E=2.1 \times 10^{5} \mathrm{MPa}, & v=0.3, \\
\sigma_{\mathrm{y}}=300 \mathrm{MPa}, \quad n=5, & \alpha=0.1 .
\end{aligned}
$$

The traction is proportionally increased from zero up to the value $t_{\max }=100 \mathrm{MPa}$. Figure 3 shows the strainenergy density as a function of the normalized arc length parameter $s / s_{0}$ along the notch path. The notch root $C$ is located at $s=0$, while $s=s_{0}$ corresponds to the point $D$. The black solid lines correspond to the strain-energy density $W$ of the plates for the Ramberg-Osgood material. The gray broken lines illustrate the solutions $W_{\circ}$ of the linear comparison problem. The strain-energy density is calculated as

$$
W_{\circ}=\frac{1}{2} \sigma_{\circ} \cdots \varepsilon=\frac{1+v}{3 E} \sigma_{\mathrm{vM}}^{2}+\frac{3}{2} \frac{1-2 v}{E} \sigma_{\mathrm{m}_{\circ}}^{2}
$$

for the linear elastic material and

$$
W=\frac{1+v}{3 E} \sigma_{\mathrm{vM}}^{2}+\frac{3}{2} \frac{1-2 v}{E} \sigma_{\mathrm{m}}^{2}+\frac{\alpha \sigma_{\mathrm{vM}}^{n+1}}{E \sigma_{y}^{n-1}} \frac{n}{n+1}
$$

(a)

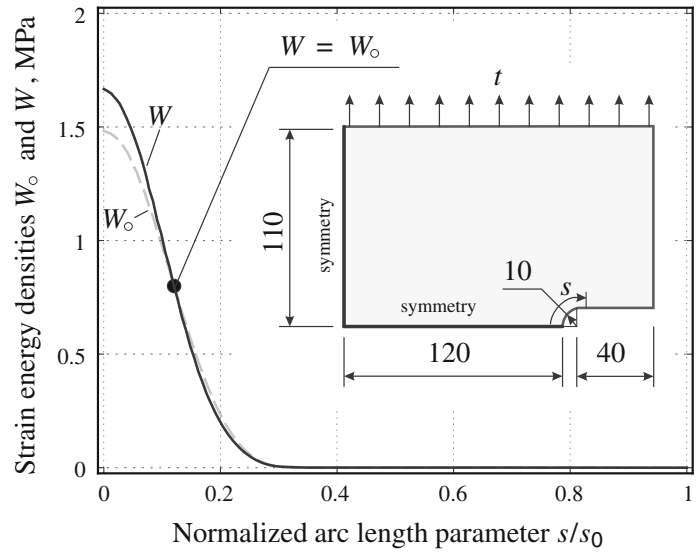

(b)

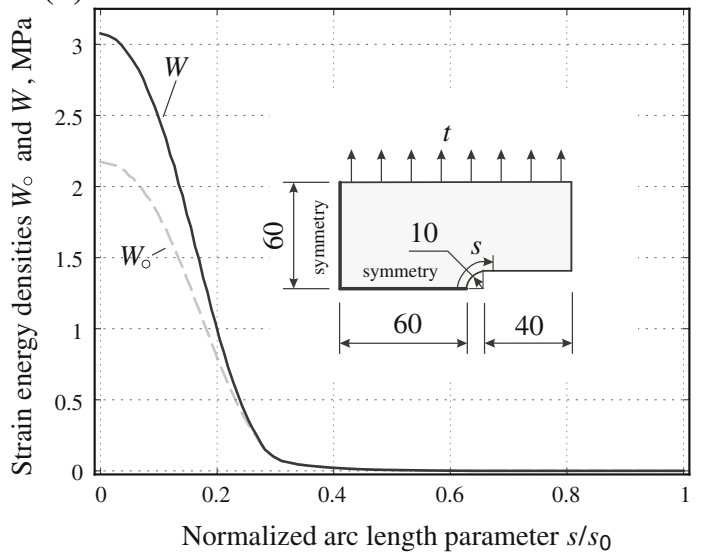

Fig. 3 Strain-energy densities for linear elastic and non-linear elastic material behavior along the notch path. a Example I, b example II 
for the Ramberg-Osgood material. From the theoretical analysis given above, it follows that if the assumptions 1 and 2 are met, then $W=W_{\circ}$ at a point along the path $C D$. As Fig. 3a shows, for the first example, such a point in a neighborhood of the notch root exists. For the second example, Fig. 3b, the strain-energy densities $W$ and $W_{\circ}$ do not coincide even far from the notch root.

Let us note that the "plastic zone" (zone, where the non-linear term in the Ramberg-Osgood material model is essential) is constrained in a neighborhood of the notch root for both the examples. However, the small "plastic zone" may affect the stress state even far from the notch root. Figure 4 shows the von Mises equivalent stress as a function of the normalized traction in the corner point $A$ of the plates. In the first example, Fig. 4a, the variation is linear that means the "plastic zone" does not affect the equilibrium far from the notch root. In the second example, Fig. $4 \mathrm{~b}$, the variation deviates essentially from the linear solution even for moderate traction values. Therefore, it is not possible to find a path $\Gamma_{\mathrm{o}}$, where the solution is linear elastic; thus, the assumption 2 is violated, and the solutions of (1) and (5) do not coincide even far from the notch root.

For the first example considered, the equality of energies in a point near the notch allows us to calculate the actual stress value. Indeed, on the free surface of the notch, the stress tensors are defined as follows:

$$
\boldsymbol{\sigma}=\sigma_{s s} \boldsymbol{e}_{s} \otimes \boldsymbol{e}_{s}, \quad \boldsymbol{\sigma}_{\circ}=\sigma_{s s_{\circ}} \boldsymbol{e}_{s} \otimes \boldsymbol{e}_{s},
$$

where $\sigma_{s s}$ and $\sigma_{s s_{\circ}}$ are the tangential stresses and $e_{s}$ is the unit tangential vector to the contour CD. Therefore

$$
\sigma_{\mathrm{m}}=\frac{1}{3} \sigma_{s s}, \quad \sigma_{\mathrm{vM}}=\sigma_{s s} \quad \sigma_{\mathrm{m}_{\circ}}=\frac{1}{3} \sigma_{s s_{\circ}}, \quad \sigma_{\mathrm{vM}}=\sigma_{s s_{\circ}} .
$$

If $\sigma_{s S_{\circ}}$ is known from the solution of the linear elastic problem, then $\sigma_{s S}$ can be calculated from (19), (20), and $W=W_{\circ}$. The equality of energies is proved only for the point in the neighborhood of the notch root, cf.
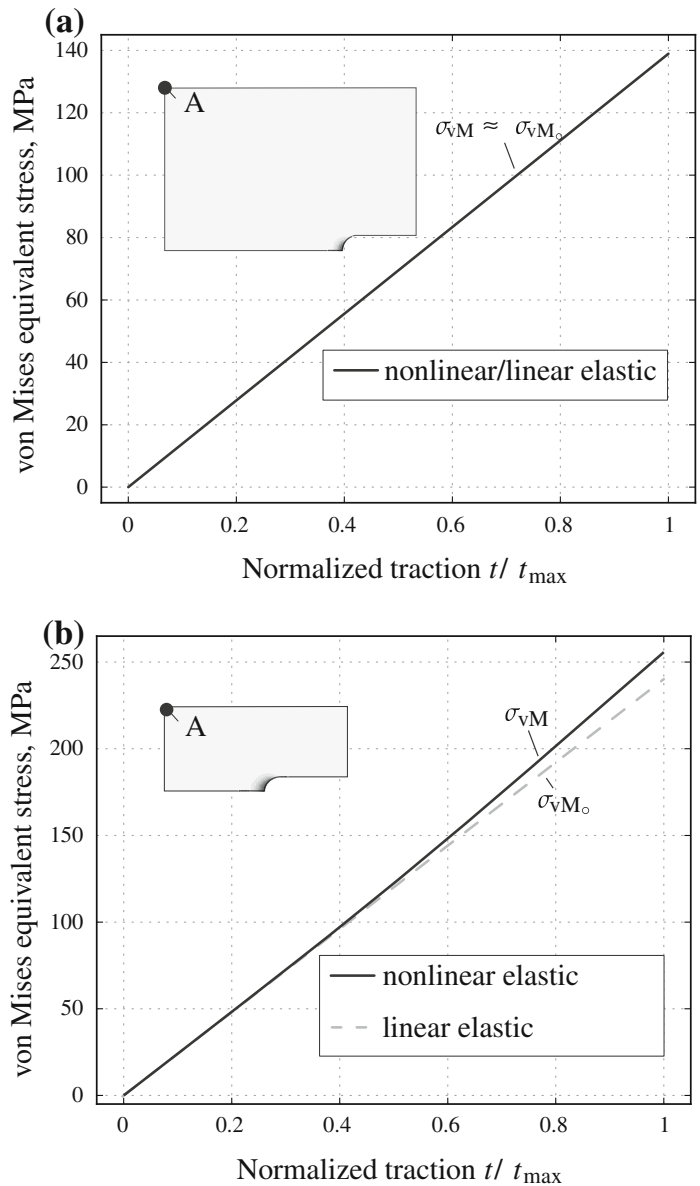

Fig. 4 Distribution of the von Mises equivalent stress over the plate face and von Mises equivalent stress vs. normalized traction in the point $A$. a Example I, $\mathbf{b}$ example II 
Fig. 3a, but not in the notch root itself. However, the position of the point will tend toward the notch root as the length of the localization path $C D$ decreases, for example by a decrease in the notch radius. The equality of the strain energies in a root of a sharp notch is assumed in [5].

In [11], notches of equal strength, i.e., notches with uniform distribution of stresses and strains along the notch boundary, are discussed. For such notches, $W=W_{\circ}$ for the whole notch path.

\section{Conclusions}

The aim of this paper was to show that approximate rules for a rapid estimation of stress fields in non-linear elastic solids near stress concentration zones such as notches, holes, etc. can be related to Eshelby-type conservation laws. In particular, applying the conservation laws, the sufficient conditions for the use of approximate methods can be established. The principal conditions are the localization of the non-linear behavior in the vicinity of the stress concentration zone, for example, in the vicinity of the notch, and the existence of a surface (in the three-dimensional case) or a curve (in the two-dimensional case) where the Eshelby tensors for the nonlinear and linear bodies almost coincide. If these conditions are satisfied, then the strain-energy density for the non-linear and linear elastic bodies coincide in some points along the notch surface. The presented examples of finite-element analyses show that these conditions not only are sufficient but also may be necessary.

\section{References}

1. Chaboche, J.L.: A review of some plasticity and viscoplasticity constitutive equations. Int. J. Plast. 24, 1642-1693 (2008)

2. Naumenko, K., Altenbach, H.: Modelling of Creep for Structural Analysis. Springer, Berlin (2007)

3. Naumenko, K., Altenbach, H., Kutschke, A.: A combined model for hardening, softening and damage processes in advanced heat resistant steels at elevated temperature. Int. J. Damage Mech. (2010), doi:10.1177/10567895103868511-21

4. Neuber, H.: Theory of stress concentration for shear-strained prismatic bodies with arbitrary nonlinear stress-strain law. Trans. ASME. J. Appl. Mech. 28, 544-550 (1961)

5. Glinka, G.: Energy density approach to calculation of inelastic strain-stress near notches and cracks. Engng. Fract. Mech. 22, 485-508 (1985)

6. Hyde, T.H., Sabesan, R., Leen, B.S.: Approximate prediction methods for multiaxial notch stress and strains under elasticplastic and creep conditions. J. Strain Anal. 40, 535-548 (2005)

7. Desmorat, R.: Fast estimation of localized plasticity and damage by energetic methods. J. Solids Struct. 39, 3289-3310 (2002)

8. Kienzler, R., Herrmann, G.: Mechanics in Material Space with Applications to Defect and Fracture Mechanics. Springer, Berlin (2000)

9. Gurtin, M.E.: Configurational Forces as Basic Concepts of Continuum Physics. Springer, Berlin (2000)

10. Maugin, G.A.: Material Inhomogeneities in Elasticity. Chapman Hall, London (1993)

11. Cherepanov, G.P.: Mechanics of Brittle Fracture. McGraw-Hill, New York (1979) 